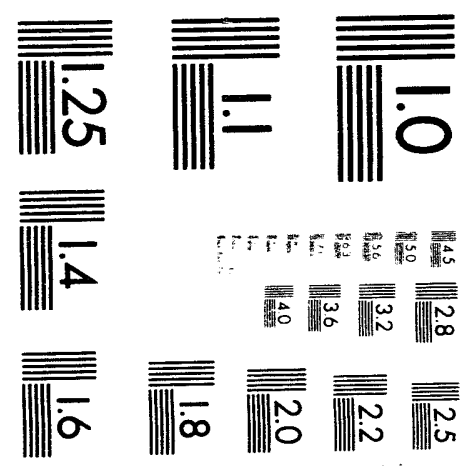



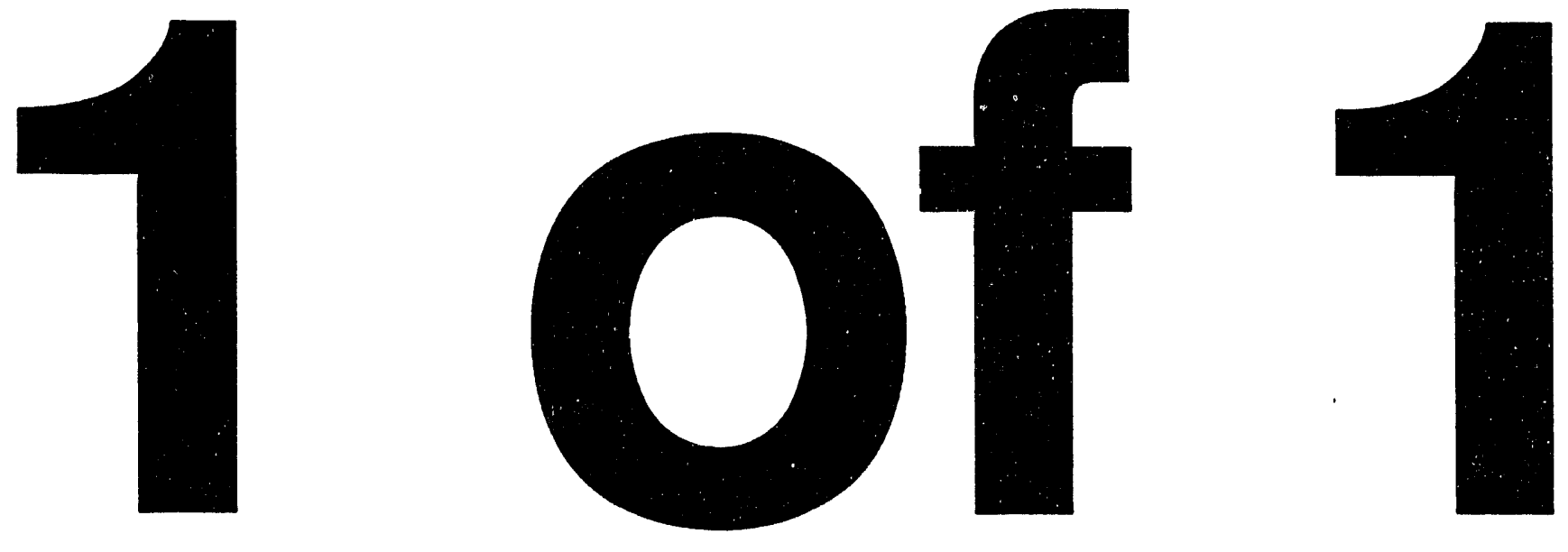


\author{
ANALYSIS AND COMPUTER TOOLS FOR SEPARATION PROCESSES \\ INVOLVING NONIDEAL MIXTURES \\ Progress Report
for Period December 1, 1992 - November 30, 1993
}

Angelo Lucia

Department of Chemical Engineering

Clarkson University

Potsdam, New York 13699-5705

July 12,1993

Prepared for

THE U.S. DEPARTMENT OF ENERGY

AGREEMENT NO. DE-FG02-86ER13552 


\section{Scope of Investigation}

This research is concerned with the development of the mathematical

- analysis, numerical analysis, and computer tools for separation processes involving nonideal, homogeneous and heterogeneous multicomponent mixtures. The mathematical analysis issues include:

(i) analysis of binary azeotropic multistage separation processes involving various product flow and composition specifications,

(ii) analysis of multicomponent, multistage separation processes involving homogeneous mixtures under various specifications,

(iii) analysis of the number of complex-valued solution to singleand multistage separation processes, including the theoretical meaning of thermodynamic states and functions,

(iv) identification of sources of and pathways to vapor-liquid solution multiplicity, and relationship of these sources of multiplicity to process attributes such as phase behavior, potential heterogeneity, stage interlinking, types of specifications, etc.

Related numerical analysis issues include:

(i) numerical analysis of existing and any newly-developed equation-tearing algorithms, particularly Rachford-Rice, sum-rates and bubblepoint methods in the real and complex domains,

(ii) numerical aralysis of equation-solving methods in the complex domain. Particular attention will focus on Newt on and various trust region methods as well as the effect of singular and saddle points on convergence.

Associated computational issues include:

(i) numerical verification and testing of any insights provided by the analyses.

(ii) comparative numerical testing of any algorithmic de wopmert. or improvements with regard to reliability and som itationa? efficiency.

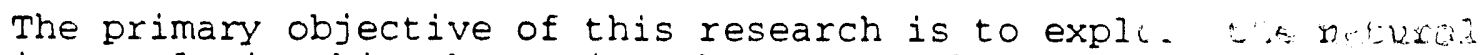
interrelationship that exists between mathematica sis sis, numerical analysis and algorithms in order to advince bt theoretical and practical understanding of the design, simusisn, operation and control tasks associated with separatiol pyosses involving nonideal mixtures.

II. Summary of Progress

Progress made during the first year of this third term of funding is organized in terms of mathematical analysis, numerical analysis and algorithmic development and is summarized below.

Mathematical Analysis

Progress in the area of mathematical analysis includes:

(i) a completion of rigorous analysis of the number of solutions to separation processes involving multicomponent homogeneous or heterogeneous mixtures and flow profile and heat duty profile specifications. 
The rigorous analysis of multistage separators involving homogeneous and heterogeneous multicomponent mixtures involving flow profile or heat duty profile specifications has been completed. It was shown that the appearcace of multiple steady-state solutions to the usual equilibrium stage model for these processes can be characterized by the determinant of the matrix of partial derivatives of total vapor (or liquid) flows with respect to stage temperatures. This fact was related to the temperature surface for bubble point liquids and, in turn, to the presence of valleys and ridges in this temperature surface. This analysis fits well within the framework originally established by sridhar and Lucia and is valid for both constant and nonconstant molar overflow conditions. Also, a first example of steady-state solution multiplicity for fixed heat duty and pressure profiles was constructed. A paper containing this material is currently under preparation.

Numerica? Analysis

Progress in the area of numerical analysis includes:

(i) study of the periodic and chaotic behavior of Newt on's method on separation-related problems in the complex domain.

(ii) extension of trust region methods for stabilizing Newton's method in the complex domain.

Many separation problems or subtasks involve the numerical solution of problems that can have more than one real solution for certain parameter values, particularly problems with potential heterogeneities. For such problems, it is well-known that Newton's method can exhibit periodic or chaotic behavior. In light of this, many numerical studies were conducted in order to build an understanding of the generic geometric structure of basins of attraction, Julia sets, the Mandelbrot sets, and the associated effects of initial and parameter values on the behavior of Newton's method in the complex domain. The particular emphasis here was placed on the determining if earlier findings for problems with a single complex variable carry over to the multivariable case. Important results in this area are that

(i) bifurcations of solutions into the complex domain are accompanied by an exchange of a pair of global minima (roots) for a local minimum (singular point) on the real domain, and a marked change in the structure of the Julia set. Rigorous proof of the fact that any singular point in the real domain is a saddle point of the norm in the complex domain was also established.

(ii) the aforementioned changes in geometric structure clearly explains why both Newt on's method and traditional trust region methods have difficulties in locating solutions of any kind, real or complex.

Using facts (i) and (ii), conventional trust region methods were extended to the cuiplex iullid in in a way that appwis to provide global convergence to a root, although rigorous proof of convergence has not been established yet.

Computational Algorithms

Progress in the development/improvement of computational methods for steady-state separation process simulation includes: 
(i) implementation and testing of an extended trust region method in the complex domain.

(ii) exploitation of the saddle point characteristics of singular points of analytic functions for improving equation-solving reliability (i.e., always finding a solution) and selectivity (i.e., finding a specific solution or all solutions).

(ii) numerical verification of various analysis results.

The proposed extension of trust region methods to the complex domain has been implemented and tested on several common subproblems in separation process simulation, including finding the roots to equations of state, dewpoint and flash problems. These numerical studies show that our extended trust region method avoids the periodic, chaotic and divergent behavior of Newton's method, and, unlike traditional trust region methods, does not terminate at singular points. Rigorous (i.e., eigenvalueeigenvector) and approximate methods of exploiting the presence of saddle points in order to ensure global convergence to solution have been developed and have performed very well in practice.

III. Compliance

Comparison of the research proposal for the funding term $112 / 92$ to $12 / 93$ ) and this report shows that the work to date is in compliance with the original agreement on which the grant was awarded.

IV. Personnel

The current grant is under the direction of Dr. Angelo Lucia, Professor of Chemical Engineering at Clarkson University. The approximate time committment for the principal investigator has been one summer month in 1991 and approximately three months during the 1990-91 academic year. The duties of the PI have been the normal administrative, technical support, and technical development duties and are expected to continue with the same relative time commitments for the remainder of the current funding period, as well as future periods of funding.

This grant has also been used as continuing support for:

(i) Mr. X. Guo

(ii) Mr. H. Li

Mr. Li is a Ph.D. graduate student in Chemical Engineering at Clarkson University and is responsible for the mathematical analysis, numerical analysis and algorithmic development in the area of homogeneous separation processes. Mr. Guo is also a Ph.D. student in Chemical Engineering and is responsible for the analysis and computational developments of separation processes involving heterogeneous mixtures. It is anticipated that both $\mathrm{Mr}$. Li and $\mathrm{Mr}$. Guo will complete all degree requirements by December 1994 .

V. Publications and Presentations

Publications

1. A. Lucia, $x$. Guo and $x$. Wang, Process simulation in the complex Domain, AIChE J., 39, 461 (1993).

Presentations

1. A. Lucia and H. Li, Multistaqe Separation Process Analysis, AIChE 
Spring National Meeting, Houston, TX, March 1993.

2. A. Lucia, Analysis and Algorithms for Multistage Separation Processes, Prairie View A \& M University, Prairie View, TX, March 1993.

3. A. Lucia, Multistage, Multicomponent Separation Process Analysis, Eastman Chemical Co., Kingsport, TN, June 1993.

4. A. Lucia, X. Guo and J.B. McLaughlin, Chaotic and Periodic Behavior of Newton's Method, SIAM Annual Meeting, Philadelphia, PA, July 1993.

Presentations 2 and 3 were invited presentations. 

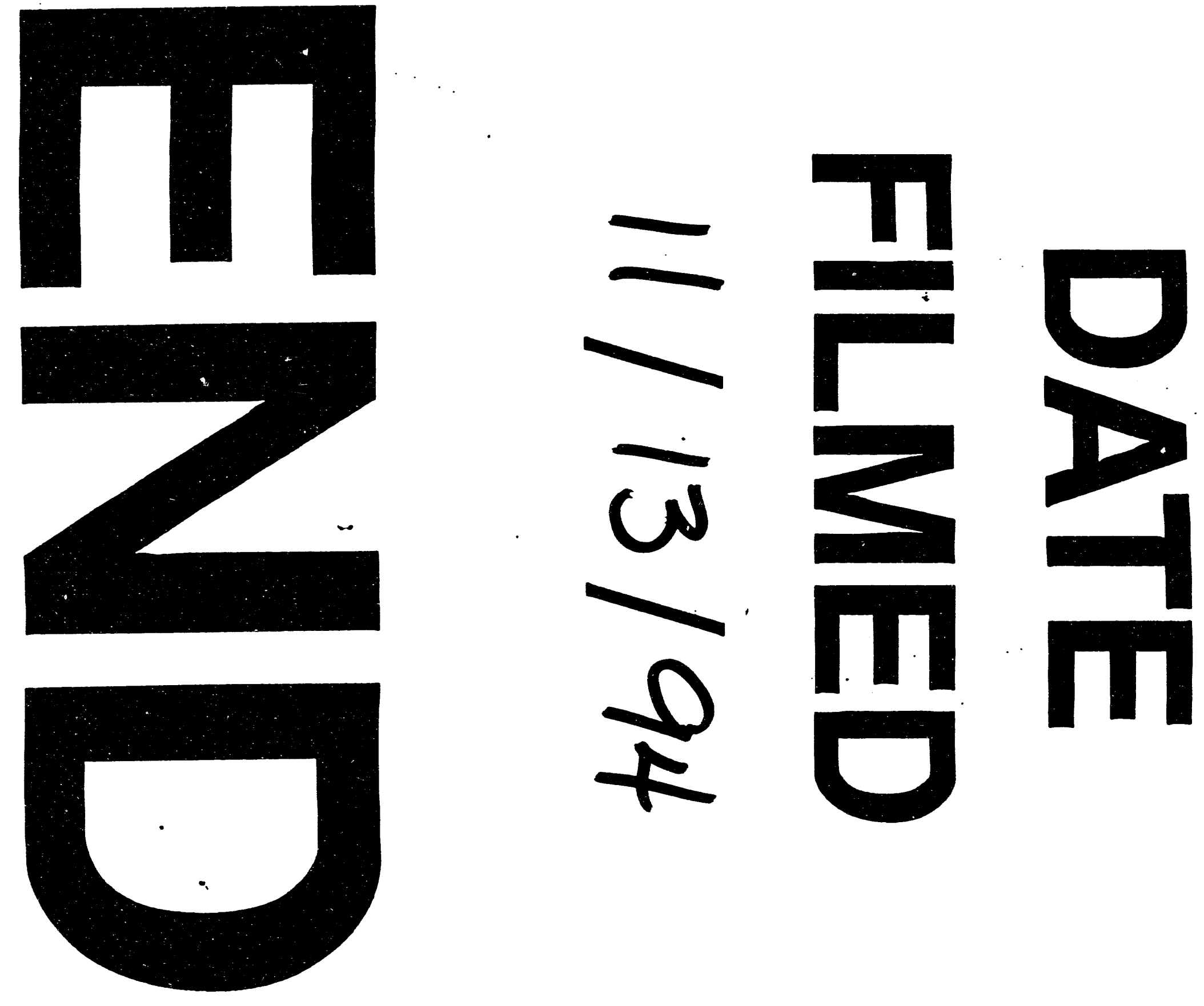
